\title{
A Socially Driven, Goal-Oriented Approach to Business Process Management
}

\author{
Mohammad Ehson Rangiha \\ Centre for HCID, City University London, \\ UK
}

\begin{abstract}
Over the recent years, there has been much discussion about the concept of Social Business Process Management (SBPM) and how it is able to overcome some of the limitations of the traditional BPM systems. This paper aims to address gaps in social BPM research by working towards a goaldriven SBPM meta-model that seamlessly integrate the process design and enactment stages. This approach also makes use of a process recommendation system to guide the activities of the users based on their social behavior and social goals. We argue that this approach will lead to truly social driven process enactment environments.
\end{abstract}

Keywords-BPM; Social BPM; Goal-Based Modeling; Social Goals; Process Goals

\section{INTRODUCTION}

Business Process Management (BPM) provides a platform for the management, measurement and improvement of business processes [2]. BPM has evolved over the years and an attempt has been made towards integrating social elements into the BPM lifecycle, although these discussions are still at their infancy [3].

Social BPM is when the essential elements of social software has been applied to the different stages of BPM. This is to effectively optimize and improve the efficiency of the traditional BPM systems. [20] defines social BPM as a "methodology for bringing more and diverse voices into process improvement activities".

Recent research has started to introduce elements of social software into the BPM lifecycle [1][3][5][7][13].

This paper aims to present the research which has been carried out so far in the area of social BPM, identify the gap in the area which is related to social BPM during enactment, and finally to propose a goal-driven model which is guided by the social user behavior in order to overcome the limitation of the traditional BPM model and to contribute to the research in social BPM.

The paper is structured in the following way: firstly some basic concepts of BPM are explained and a number of limitations of the current BPM model are presented (Section 2).

In Section 3, social software is discussed. Section 4 overviews social BPM, while in section 5, goal-based modeling has been proposed as a way forward in social BPM. In Section 6, a proposed recommendation mechanism in the context of user's social behavior is discussed. Finally in

\author{
Bill Karakostas \\ Centre for HCID, City University London, \\ UK
}

Section 7, a conclusion is drawn from what has been discussed and the way forward for future research is proposed.

\section{Business Process Management}

\section{A. Overview of $B P M$}

BPM is a field of knowledge where IT and management meet [9] and it "includes concepts, methods, and techniques to support the design, administration, configuration, enactment, and analysis of business processes" [10].

There are a various models regarding the overall BPM lifecycle, however for the purpose of our research we have adapted the model proposed by Aalst et al [12].

According to van der Aalst et al. [12], the BPM lifecycle consists of four main stages: process design, implementation, process enactment and finally process improvement/evaluation.

\section{B. Limitations of $B P M$}

The current state of BPM practice has a number of limitation which some of them are listed below [14]:

Lack of Information Fusion: The traditional BPM model follows a top-down approach where processes are designed and imposed on the users to follow [13] [23].

Model- Reality divide : Users not having a say in the process design stage results in them not using the processes; this in turn creates a gap between what the process actually is and what happens in real life [4][13][23].

Information Pass-On Threshold and Lost Innovation: Valuable feedback from users regarding the processes remains unused due to hierarchical controls which can prevent sharing of knowledge [4][13].

Strict Access-Control: In traditional BPM systems strict access control for the users is quite common and only actors which have been chosen and given specific access are allowed to execute them [11].

Lack of Context: The BPM engine focusses on controlling the flow and interaction of the data independent of the rest of the system. Therefore, the contexts of each case in which the data are appearing or the transactions are taking place are not easily accessible [16]. It is seen that majority of the business processes in one way or another interact with their surrounding environments, hence it is important to have an understanding of the context in which an activity or process is taking place in order to be able to improve them. 


\section{SOCIAL SOFTWARE}

"Social software is a general term encompassing a set of tools and applications that enable group interaction and computer-mediated communication"[17]. Today, the trend of growth in the usage of social software in different platforms is increasing rapidly. We argue that social software concepts can be adapted in order to improve and overcome some of the challenges found in the current business process management systems.

The fundamental elements of social software is enabling interaction and maximizing participation between users and communities. Some of these have been mentioned in [15], listing the following characteristics: Weak Ties (allow spontaneous creation of contacts between non-predetermined individuals [19]), Social Production (Enable innovative and unexpected contributions and feedback regardless of geographical location [17] [1]), Egalitarianism (The maximization of participation through the role of trust and the equal rights that the users have to contribute to eventually reach the best practice [19]), Mutual Service Provisioning (The transformation of the organization's model from a oneway service system to a service-exchange system [19]. This results in the customer having a say in the design of the products and services they receive).

\section{Social Business Process Management (SBPM)}

\section{A. Overview of SBPM}

Social BPM is the intersection of social software and BPM to overcome some of the limitations of the traditional BPM systems. Social BPM is a "methodology for bringing more and diverse voices into process improvement activities" and it describes as collaboratively designed and iterated processes [20].Majority of the definitions given about SBPM until now mostly emphasize the role of collaboration during the process design stage of the BPM lifecycle and neglect how the SBPM engine could work during runtime.

SBPM offers a platform that enables contribution from various users of the community during all the stages of the BPM lifecycle. This increased participation ensures the users actually follow the processes during enactment; this overcomes the model-reality divide limitation which has been identified in the traditional BPM systems. However what has been discussed in SBPM is minor contributions of social software to BPM and not a framework explaining what the different elements of the SBPM are.

Therefore there is much to explore in order to exploit and fully benefit from the integration of BPM lifecycle and social software [3] [4]. BPM analysts believe that BPM application developers are socializing, not the end users themselves [8]. So in other words, based on the current understanding of SBPM, the developers are using social software in order to improve their experience of BPM and this ignores runtime social collaboration [15].

Social features not only play an integral role in the design of the processes, but the BPM lifecycle also benefits from these features during the execution of the processes. [22].
Adapting a SBPM framework not only needs a fully functional and validated model for it to be adaptable in an organization, but rather the organization also needs to be prepared to adapt to a whole new way of approaching support [27].

\section{B. The Potential and Limitations of SBPM}

There a number of potential benefits which SBPM can produce, some of these include as they have been elicited in [14]: exploitation of weak ties and implicit knowledge [1][3] (allowing the discovery and capturing of tacit and informal knowledge), transparency [3][5] (increase of procedure visibility in the organization), decision distribution [3][16]: (more well-informed and collective decision making), knowledge sharing [3][28][5] (allowing easier dissemination of knowledge).

Social software and traditional BPMS have different characteristics due to which their integration can also bring about a number of disadvantages and raise some challenges for the business. Potential limitations of SBPM have also been identified which include: learning effort [4][1] (major cultural shift required in the organization), security [4][5] (access rights given to many people can create an insecure environment), quality [4] (the quality of the content could be reduced as input is received from different people), difficulty to evaluate [5] (due to scattered benefits of SBPM it is difficult to evaluate them), process management (due to the flexible nature which exists it becomes a challenge to manage the interaction and overall processes)[14].

\section{SBPM at Process Design and Enactment}

There has been much discussion about how BPM can benefit from social software during the process discovery stage. In SBPM, users are all involved in the modeling of the processes [1], unlike the current approach in traditional workflow systems.

Brambilla et al. [7] have proposed an extension of the BPMN which includes using features of social software; it consists of various technologies and tools in order to include the contributions of the users from the outset of the process design stage. Although the idea of collaborative input at the design stage of the processes has been used previously by the Scandinavian School [28] in the 1970s.

Overall, although this approach eases the process of design and allows a wider collaboration from the users, however it does not remove the sequential and rigid nature of the traditional BPM systems which is the root cause for many of its limitation. Thus, this extension would not lead to a truly socially driven SBPM framework.

Kemsley [1] defines enactment or runtime collaboration as the modification of the processes during execution. This includes catering for unplanned participation of the users during the runtime of the processes. However no research in the area of social BPM, to date, has proposed a framework to

For SBPM [15]. Therefore this is the main area in which further research needs to take place and a clear way forward to overcome the sequential nature of the processes in BPM to be proposed. 


\section{FURTHER RESEARCH INTO SBPM}

\section{A. Goal-Oriented Modelling}

Goal-oriented business process modeling [32], strives to document the intention and goals behind the activities. Goals are defined as objectives that should be achieved by the system and its environment [34]. Goals are also commonly used in requirements engineering to ascertain the state which the user would like to reach after performing a specific activity [33].

Most of the research about social BPM mainly revolves around SBPM in the design stage. Adapting the approach of goal-based modeling and following this structure during the enactment stage, will also affect the design stage of the BPM lifecycle and it would need to adapt the proposed goaloriented modeling approach [15].

As mentioned by Kemsley [1] social BPM may change the very nature of process modeling, so depending on the approach taken during the enactment, the process design approach might also need to be changed.

The defined goals may have sub-goals which need to be accomplished in order to fulfill the main goals [1] [24].This is in contrast to the traditional procedural based BPM systems [24] where performers are bound to follow a set of predefined rigid series of steps.

This approach would produce the desired flexibility and would be a platform for collaborative process discovery through defining end goals. The more the goals and sub-goals are defined in detail, the more robust and rigid the workflow, while the fewer and more general the goals, the more flexibility is given to the user to come up with their own set of activities. This heavily depends on the nature of the process, but in general cases, when there are many goals for a relatively small scenario, it is an indication that there is limited flexibility during the execution of the processes[15].

When the goals are fewer and are defined in a more general manner, it allows for a higher degree of flexibility and dynamic behavior for the user(s).

The priorities of the goal/sub-goals set, need to be in line with the general policies and aims of the company. These goals could be re-evaluated, subject to collective approval based on the suggestions for improvements received from the wider community and users. The continuous engagement of the end users and clients with the system and processes would also allow more insightful feedbacks from the customers which could be used to enhance the goals and processes [15].

Figure 1 presents a visual overview of our proposed goal driven SBPM process, out of which we plan to derive a metamodel.

Goal-based modeling is a potential approach in order to overcome the limitations of the traditional BPM models and propose a comprehensive model for social BPM where a dynamic and social behavior is visible in all the stages of the BPM lifecycle.

\section{B. SBPM at Enactment}

In most workflow management systems, users are expected to execute the predefined tasks in a specific order and sequence. When it comes to SBPM, this traditional approach is not in harmony with the flexible characteristics found in social software. Social BPM should enable the collaborative modeling and execution of processes with a certain degree of flexibility. In social BPM, the flow and sequence of actions should not be enforced upon the user, but rather it should allow and support the enactment of the processes, exploring the sharing of knowledge and the business best practices [5].

According to Brambilla et al. [6] participatory enactment transfers this collaboration in the process design stage to the execution and runtime of the processes. This is because the processes are designed on the flyer at the time of their execution therefore there is a seamless integration of the design and execution stage of BPM lifecycle.

By adapting a goal-based modeling approach in the process discovery stage of the BPM lifecycle, during the enactment of the processes, the users will have a certain degree of flexibility and freedom when executing the processes. On the one hand, the goals defined in the design stage act as controls and provide direction to the sequence of activities which need to be performed, and on the other hand this will leave an unstructured space for the user to decide their own course of action in order to accomplish the particular goals [15]. Figure 1 shows how the goal is explicitly mentioned, but how to achieve them is simply recommended by the system through different alternatives, and the user is free to decide how the tasks are executed. 


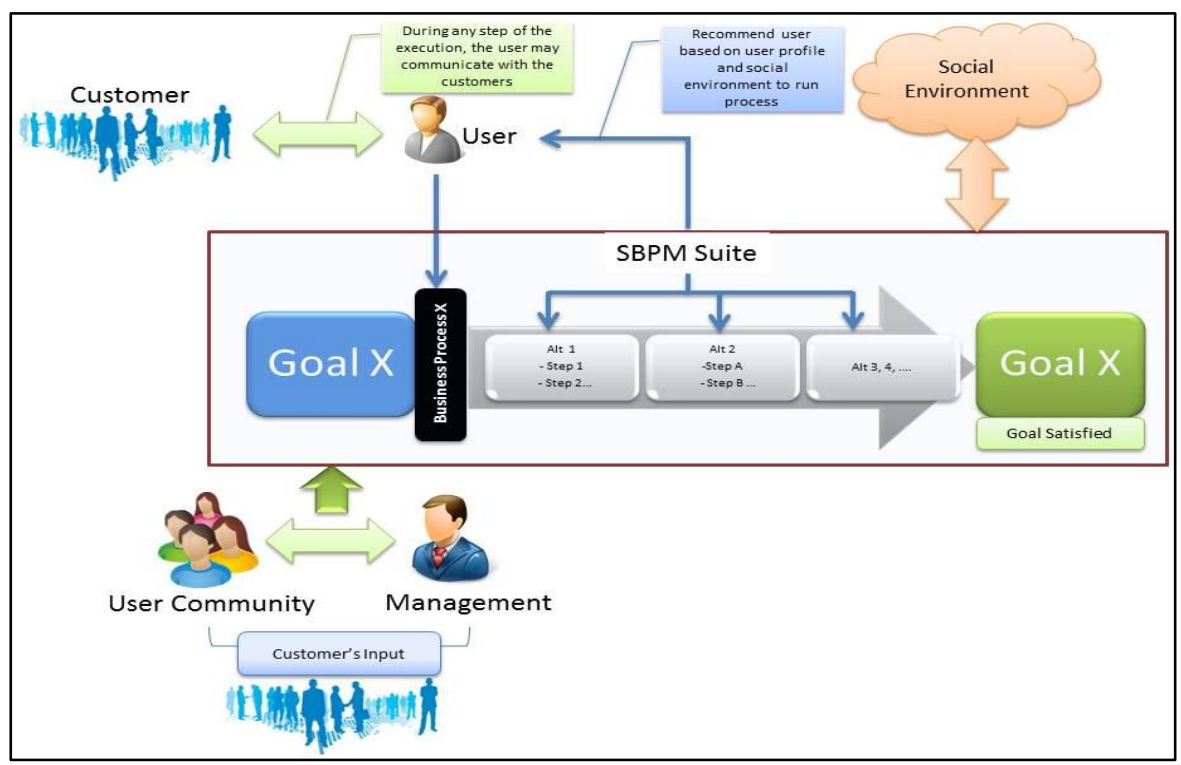

Fig. 1. Goal-Driven Social BPM Interaction Model

\section{Socially Guided Business Process Modelling}

The model in figure 2 illustrates the different elements of the model which are explained in this section. This is a high level view of how these features are linked to one another and further research needs to be carried out in order to explore in more detail how they will function in practice.

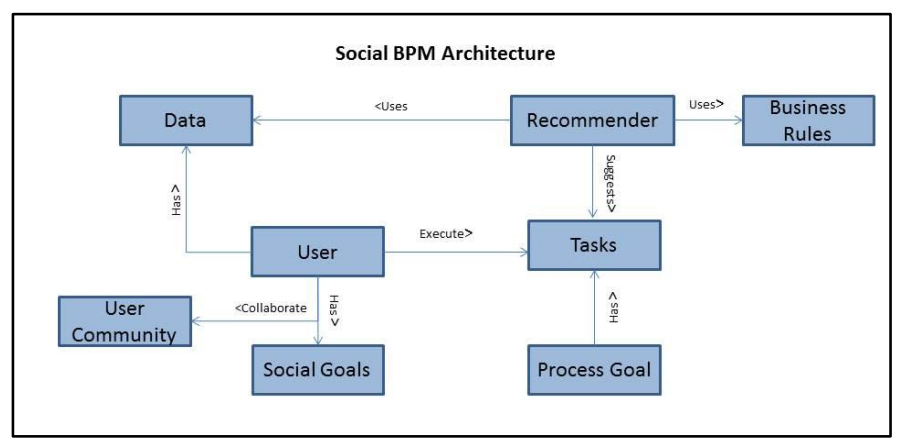

Fig. 2. Social BPM Architecture

\section{A. User-System Interaction through Recommendation}

In order to achieve a specific goal, the user is flexible to choose the course of action he chooses. This however is guided by the system in order to firstly ensure the steps taken lead to a state or goal which is expected, and secondly, so that the steps taken can be monitored, captured and re-used by the Social BPM suite in the future. The system can make process /step recommendations based on the data gathered from the user. This data would determine the suitability and interest of the user to be engaged in a process to achieve a given goal. The process recommendation system [26] based on this data interacts with the user in order to achieve the sub-goals which are needed in fulfilling the overall goal.

The concept of recommender systems have extensively been used previously in e-commerce, however they have not been utilized in the context of process/task recommendations.
For the purpose of the SBPM framework, recommendations based on Hybrid approach would be most suitable. This is because it combines recommendations that have been made in the past (content-based recommendations), with recommendations made by other users with similar tastes and preferences (collaborative recommendations)[18].

During this interaction, the user is given a certain level of flexibility to insert or remove steps into the processes. This can involve, for example, eliminating a certain step by proposing and achieve a sub-goals through a different route which might be better than the one suggested by the system. In this context, flexibility here is "the ability to adapt the process flow on demand through adding, skipping, or sequence reordering of process steps" [21].

Figure 1 captures this concept, by illustrating how the goals are set initially, and then based on them, how the system can make recommendations. Users then have the option to follow the recommendation or choose an alternative step.

\section{B. Business Rules}

Constraints and dependencies are from the essential elements of recommendation mechanism. Because before the recommender assigns or suggests a tasks, it should be guided by a number of business rules which capture any possible dependencies of the tasks. In other words, the sub-goals are recommended in such a fashion that their pre-requisites have already been fulfilled before the recommendation. Otherwise what is suggested may not be executable by the user or the system as there are one or more dependent tasks which need to be satisfied first.

\section{Social Goals}

As the proposed model is based on a goal-oriented approach, it is important to specify what type of goal is referred to when discussing goals in the context of social BPM. 
The topmost goals are process goals, which are dependent on the cases study and specific scenarios. These might have various sub-goals which need to be fulfilled in order for them to be satisfied.

The other types of goals in this model are social goals and relate to the user/customer and their motivation for getting involved in a particular process. These goals can vary from person to person, and can belong to categories such as social

affiliation goals, social responsibility goals and social concern goals [25].

\section{Conclusion And Future Research}

In conclusion, SBPM research is at an early stage and there is still lack of an effective method for applying the social elements to the enactment stage of BPM. The solution proposed in this paper is in terms of working towards a metamodel of the social BPM lifecycle that applies goal-oriented modeling principles to the design and enactment stages. With the use of goal-based modeling we are able to make enactment more flexible and allow collaborative participation of the processes.

This flexibility, however, needs to be controlled, so the enactment does not divert from its main path and from achieving its end goal. According to Ramdas [31] there are different degrees of flexibility found when it comes to the interaction of the processes:

1) Ad hoc/Unstructured: there is no ordered workflow, decisions and actions are performed on the fly and these processes are normally manually executed and consist of unstructured activities.

2) Structured: These types of processes are well-defined with the performers and series of activities predefined.

3) Collaborative: these processes include on the fly interactions, however within a certain defined boundary and framework.

Our research, in particular addresses the collaborative aspect by proposing a goal-based approach. A similar sort of categorization has also been proposed by Brambilla et al [30] ranging from a closed BPM system which the tasks are highly rigid and actors all predefined, to the process mining category where the activities are executed freely and there is no set of activities defined.

In our approach, the steps to be performed are simply suggested by the system based on the previous iterations and through a process recommendation mechanism, the user is then free to either accept or reject the proposed step. It is important to note that the sub-goals required to satisfy the overall goal of the process must be fulfilled by the user. This gives the required flexibility to the user and at the same time preserves the integrity of the system to ensure the process and steps are moving in the right direction.

The recommendations are based on the data gathered from the user to ensure they have an interest in the process they are recommended to be involved. Other users who also have interest in the same processes can participate in achieving the recommended tasks.
Goal-based modeling has of course, its own limitations that need to be addressed before its adoption for social BPM. One of the most important challenges in defining the goals in this model is dealing with conflicting priorities between goals and identifying the goals themselves and not confusing them with the steps involved in accomplishing the goals [29].

In summary, this paper has focused on the current stage of our on-going research in the area of social BPM with a special emphasis on the process discovery and enactment stage and how socially guided BPM can change both the enactment stage of the BPM lifecycle as well as the process discovery stage as there is seamless integration of these two stages.

In future research we plan to formalize a metal-model for the goal-oriented SBPM, and to apply this model to a case study in order to validate our proposed approach. Real goals will be used from a case study and the meta-model presented in this paper will be apply to the scenario. This, through an evolutionary processes will lead to a standardized meta-model for a socially enabled BPM system. The different elements of the recommender system also needs to be explored further to provide a comprehensive framework for a socially driven, goal-based BPM model.

\section{REFERENCE}

[1] Kemsley, S.: Leveraging social BPM For Enterprise Transformation: In Social BPM Work, Planning and Social Collaboration Under the Impact of Social Technology, Swenson, KD., Palmer, N., et al.: BPM and Workflow Handbook Series, pp. 77 - 83, Future Strategies Inc, USA (2011).

[2] R.G. Lee, B.G. Dale, "Business process management: a review and evaluation", Business Process Management Journal, Vol. 4 Iss: 3, pp.214 - 225, MCB UP Ltd (1998).

[3] Brambilla, m., Fraternali, p., Combining social web and BPM for improving enterprise performances: the BPM4People approach to social BPM, In Proceedings of the 21 st international conference companion on World Wide Web, pp. 223-226 ACM Publishers, New York (2012).

[4] Filipowska, A., Kaczmarek, M., Koschmider, A., Stein, S., Wecel, K., Abramowicz, w., Social Software and Semantics for Business Process Management - Alternative or Synergy?, in Journal of Systems Integration, vol 2, No 3, pp.54 - 69 (2011).

[5] S. Erol, M. Granitzer, S. Happ, S. Jantunen, B. Jennings, A. Koschmider, S. Nurcan, D. Rossi, R. Schmidt, P. Johannesson. Combining BPM and Social Software: Contradiction or Chance? In Journal of Software Maintenance and Evolution: Research and Practice, vol. 22 pp. 449-476, John Wiley \& Sons, Ltd (2010).

[6] Brambilla, M., Fraternali, P., Vaca, C.: A Notation for Supporting Social Business Process Modeling Third International Workshop, BPMN 2011 Proceedings, vol. 95, pp. 88-102, Springer Berlin Heidelberg (2011).

[7] Brambilla, M., Fraternali, P., Vaca, C.: BPMN and Design Patterns for Engineering Social BPM Solutions: In BPM 2011 International Workshops, vol. 99, pp. 219-230, Springer Berlin Heidelberg (2012).

[8] Swenson, K.: Who is Socializing in Social BPM? 12 May 2010 [Blog Entry], Collaborative Planning \& Social Business. Available: http://social-biz.org/2010/05/12/who-is-socializing-in-social-bpm-2/ [Accessed Dec 2013].

[9] Filipowska, A., Kaczmarek, M., Kowalkiewicz, M., Zhou, X., Born, M., Procedure and guidelines for evaluation of BPM methodologies: In Business Process Management Journal, vol. 15 Iss: 3, pp.336 - 357. (2009).

[10] Weske, M.: Business Process Management, Concepts, Languages, Architectures, Springer-Verlag Berlin Heidelberg (2007).

[11] Statistics about Facebook users, http://www.checkfacebook.com/ [Accessed on 1st Feb 2013] 
[12] Van der Aalst, W.M.P.: Business process management: a personal view: In Business Process Management Journal, vol. 10, no.2, pp. 744-791 Emerald Group Publishing (2009).

[13] Schmidt, R., Nurcan, S.: BPM and Social Software: In BPM 2008 International Workshops, vol 17, pp 649-658, Springer Berlin Heidelberg (2009).

[14] Rangiha, ME., Karakostas, B.: Towards a Meta-Model for Goal-Based Social BPM. (In press) In: Post-Proceedings of Lecture Notes in Business Information Processing (LNBIP) series, Springer, 6th BPMS2 workshop in conjunction with BPM 2013 conference, Beijing, China (2013).

[15] Rangiha, ME., Karakostas, B.:Goal-Driven Social Business Process Management. In: Proceeding indexed by IEEE Xplore and various international databases, to be published by The Science and Information Organization, Science and Information (SAI) Conference Proceedings, pp. 894 - 901, London, UK (2013).

[16] Van der Aalst, W.M.P., Weske, M., Gr"unbauer, D.: Case Handling: A New Paradigm for Business Process Support: In Journal Data \& Knowledge Engineering, vol.53, issue.2, pp. 129 - 162, Elsevier Science Publishers, The Netherlands (2005).

[17] Abbattista, F., Calefato, F., Gendarmi, D., Lanubile, F.: Incorporating Social Software into Agile Distributed Development Environments: In proceedings of the 23rd Automated Software Engineering (ASE) workshop, pp.46-51 (2008)

[18] Burkhart, T., Weis, B., Werth, D. and Loos., P.: Towards ProcessOriented Recommender Capabilities in Flexible Process Environments -
State of the Art: 45th Hawaii International Conference on System Sciences (HICSS), USA, Hawaii (2012).

[19] Bruno, G., Dengler, F., Jennings, B., Khalaf, R., Nurcan, S., Prilla, M., Sarini, M., Schmidt, R., Silva, R.: Key challenges for enabling agile BPM with social software: In Journal of Software Maintenance and Evolution: Research and Practice 23, 297-326 (2011)

[20] Richardson, C., Is social BPM a Methodology, A Technology, Or just a lot of Hype? 20 May 2010 [Blog Entry]. Forrester. Available: http://blogs.forrester.com/clay_richardson/10-05-20social_bpm_methodology_technology_or_just_lot_hype[ Accessed Dec 2013] .

[21] Dorn, C., Burkhart, T., Werth, D and. Dustdar, S. : Self adjusting Recommendations for People-Driven Ad-Hoc Processes: in Business Process Management. pp. 327-342: Springer, Berlin / Heidelberg (2010).

[22] R. Schmidt, F. Dengler, and A. Kieninger.: Co-creation of value in IT service processes using semantic mediawiki: In BPM 2009 International Workshops, v.43, pp. 255-265. Springer Berlin Heidelberg (2010).

[23] Palmer, N.: The Role of Trust and Reputation in Social BPM: In Social BPM Work, Planning and Social Collaboration Under the Impact of Social Technology, Swenson, KD., Palmer, N., et al.: BPM and Workflow Handbook Series, pp. 35-43, Future Strategies Inc, USA (2011).

[24] Pucher, MJ.: How to link BPM Governance and Social Collaboration through an Adaptive Paradigm: In Social BPM Work, Planning and Social Collaboration Under the Impact of Social Technology, Swenson, KD., Palmer, N., et al.: BPM and Workflow Handbook Series, pp. $57-$ 75, Future Strategies Inc, USA (2011) 